\title{
Magnetic Resonances of Protons in Ethyl Alcohol*
}

\author{
JAMES T. ARnold $\dagger$ \\ CERN, Geneva, Switzerland \\ (Received September 20, 1955)
}

\begin{abstract}
Studies have been made of the proton magnetic resonances of ethyl alcohol under considerably higher resolution than has heretofore been reported. A description of the apparatus includes details of construction of a large permanent magnet, with some of the considerations directed toward improvement of resolution by reduction of field inhomogeneities. Also, the features of a specially designed nuclear magnetic resonance head are described. This head is provided with facilities for spinning the sample for the further reduction of the effective field inhomogeneity. Considerations of stability of the field and the radio-frequency components are briefly discussed.

Recordings of the observed spectra of ethyl alcohol are shown and are related to the predictions of calculations based on the use of a Hamiltonian which includes a particularly simple form of spin-spin interaction. The features of the spectra are finally resolved to about $\frac{1}{2} \mathrm{cps}$ in $30 \mathrm{Mc} / \mathrm{sec}$, and the agreement between predicted and observed structures is reasonably good.

The spectra were found to be strongly modified by the presence or almost complete absence of contamination of the ethyl alcohol by $\mathrm{H}^{+}$or $\mathrm{OH}^{-}$ions. The effect is attributed to an exchange process involving the hydroxyl proton of the ethyl alcohol molecule. The characteristic times between exchange events have been approximately determined for specified concentrations of $\mathrm{H}^{+}$and $\mathrm{OH}^{-}$ions up to the order of $10^{-5}$ normal.
\end{abstract}

\section{INTRODUCTION}

$\mathrm{F}^{\mathrm{o}}$ OR several different reasons, nuclear magnetic resonances may exhibit structures which have particularly fine details in the case of many liquids. This paper describes the development of apparatus capable of the high resolution necessary to the study of proton magnetic resonance spectra in organic liquids and reports the observation and interpretation of the spectrum of ethyl alcohol.

For large applied fields, a bare nucleus exhibits a magnetic resonance whose characteristic angular frequency is given by $\omega=|\gamma| H_{0}$, where $\gamma$ is the gyromagnetic ratio of the nucleus and $H_{0}$ the magnitude of the applied field. In the samples used in nuclear magnetic resonance experiments, the nuclei are not bare, and it has been recognized for some time that the electrons in the microscopic environment of the nuclei produce small displacements of their resonance frequencies. ${ }^{1}$ Since the distribution of the valance electrons is influenced by the chemical bonding of an atom, it is to be expected that the displacement of the nuclear magnetic resonance frequency will depend upon the chemical compound of the element investigated. The dependence of the nuclear magnetic resonance frequency on the distribution of the electrons is called the "chemical shift" and has been observed by several investigators..$^{2-4}$

In a chemical compound which contains two or more otherwise identical nuclei at chemically nonequivalent

\footnotetext{
* Work done in partial fulfillment of the requirements for the degree of Doctor of Philosophy at Stanford University. This research was supported in part by grants from the Office of Naval Research and the U. S. Atomic Energy Commission.

$\uparrow$ Present address: Stanford University, Stanford, California.

1 W. E. Lamb, Phys. Rev. 60, 817 (1941).

2 W. G. Proctor and F. C. Yu, Phys. Rev. 77, 717 (1950)

3 W. C. Dickenson, Phys. Rev. 77, 736 (1950).

${ }^{4}$ H. S. Gutowsky and R. E. McClure, Phys. Rev. 81, 276 (1951).
}

sites, the different distributions of valence electrons leads to the possibility of an "internal chemical shift." This internal chemical shift was first revealed through the phenomenon of spin-echo modulation discovered by $\mathrm{Hahn}^{5}$ in many liquids. Hahn and Maxwell in subsequent papers $^{6}$ showed that the spin-echo modulation consisted of field-dependent and field-independent parts. In their interpretation, the field-dependent part was attributed to the internal chemical shift, while the field-independent part was explained by the introduction of a rotationally invariant interaction between nuclear spins which is independent of the applied field and is called "spin-spin interaction." This same interaction was independently discovered by Gutowsky et al. ${ }^{7}$ at about the same time.

The modulation of the spin-echo envelope, observed in the case of molecules containing chemically nonequivalent nuclei, indicates that there must be a corresponding resolvable structure in the magnetic resonances of the nuclei. Structures in the spectra of such molecules, which consist of field-dependent chemical shifts and field-independent spin-spin splitting of lines, have indeed been observed. The partial resolution of the magnetic resonance spectra of protons in organic compounds was first reported by the author with Dharmatti and Packard, ${ }^{8}$ for several of the alcohols. In subsequent investigations, the spectra of numerous organic molecules were examined under slowly improving resolution. Much of the effort was directed toward the further study of the ethyl alcohol molecule.

As a representative organic compound, ethyl alcohol is particularly well suited to the study of proton mag-

\footnotetext{
5 E. L. Hahn, Phys. Rev. 80, 580 (1950).

${ }^{6}$ E. L. Hahn and D. E. Maxwell, Phys. Rev. 84, 1246 (1951);

E. L. Hahn and D. E. Maxwell, Phys. Rev. 88, 1070 (1952).

${ }^{7}$ Gutowsky, McCall, and Slichter, Phys. Rev. 84, 589 (1951).

8 Arnold, Dharmatti, and Packard, J. Chem. Phys. 19, 507 (1951).
} 
netic resonance spectra. In the first place, its protons, like those in many other organic liquids, exhibit relaxation times of the order of one second, corresponding to extremely small natural line widths of a fraction of a cycle per second. It is this feature which allows the study of very fine structures of the spectrum, and which thereby has motivated the efforts directed to the development of apparatus capable of comparable resolution. Another advantage of the use of ethyl alcohol in this study lies in the fact that the splitting due to the spin-spin interaction, while sufficiently large in comparison to the resonance line widths, is at the same time smaller by a factor ten than the differences of the internal chemical shifts in a field of 7000 gauss. This circumstance brings about a grouping of the lines which permits a simple interpretation of increasingly finer structures with increasing resolution in a one-toone correspondence with successive approximations, in powers of the ratio of spin-spin splitting to differences of internal chemical shift, of calculations based on a perturbation method..$^{9,10}$ The interpretation of the spectrum is thus greatly simplified, since, in the general case, the assignment of resonance lines to particular transitions is often not obvious.

\section{APPARATUS}

The apparatus used is of the crossed coil type. ${ }^{11,12}$ Throughout this discussion, the nomenclature employed by Bloch will be used. In the nuclear induction "head" a transmitter coil establishes an oscillating field of amplitude $2 H_{1}$ in the $x$-direction of the coordinate system. A receiver coil surrounding the sample placed in this oscillating field has its axis in the $y$-direction so as to be decoupled from the transmitter coil. A large steady magnetic field is applied in the $z$-direction with a magnitude $H_{0}$. Observations were carried out by slowly varying $H_{0}$ and recording the various lines of the spectra, each line appearing when $H_{0}$ has the appropriate value to render its frequency equal to the angular frequency $\omega$ of the oscillating field. The phase of the receiving system was adjusted to detect the pure absorption ("v-mode") and $H_{1}$ was chosen small enough to avoid saturation of the resonances. Furthermore, the rate of change of $H_{0}$ was sufficiently slow as to avoid transient effects by establishing the conditions of "slow passage" through resonance. ${ }^{11,12}$

In order to avoid saturation broadening of the very narrow lines encountered in the spectra, it is necessary that the amplitude of the oscillating field $2 \mathrm{H}_{1}$ be held extremely low. If the longitudinal and transverse relaxation times ${ }^{11}$ are equal, the lines will be unsaturated if the inequality

$$
|\gamma| H_{1}<\Delta \omega
$$

\footnotetext{
${ }^{9} \mathrm{~F}$. Bloch, preceding paper [Phys. Rev. 102, 104 (1956)], see Sec. 6.

${ }_{10} \mathrm{~W}$. A. Anderson, following paper [Phys. Rev. 102, 151 (1956)].

${ }^{11}$ F. Bloch, Phys. Rev. 70, 460 (1946).

${ }^{12}$ Bloch, Hansen, and Packard, Phys. Rev. 70, 474 (1946).
}

is satisfied, where $\Delta \omega$ is the line width in units of angular frequency. In the case of ethyl alcohol and many other organic liquids, where the line widths are a fraction of a cycle per second, this inequality demands that the oscillating field be held to values smaller than $10^{-4}$ gauss.

In a recent paper by Bloembergen and Pound, ${ }^{13}$ it was pointed out that the loading of the nuclear induction by the currents which flow in the receiver coil causes an effective shortening of the relaxation time. While, contrary to their statement, this effect would also give a corresponding broadening of resonance line widths obtained under the steady state conditions of slow passage if large filling factors were used, it contributed very little to the line widths in our experiments where the filling factor was about $7 \%$.

Particular care must be observed to preserve the conditions of slow passage in the case of narrow lines. Since the precise observation of a line whose width is $\Delta \omega$ involves in effect the determination of a frequency to within this limit, the time spent in traversing the line must be comparable to or greater than the inverse of $\Delta \omega$; the time rate of change of the sweeping field which scans the line must therefore decrease in proportion to the square of the line width observed. With the observed line widths in the proton magnetic resonance spectrum of ethyl alcohol being about one-fourth cycle per second, the sweeping rate must be held to less than 0.06 cycle per second per second, or about $1.5 \times 10^{-5}$ gauss per second. Any fluctuation of the field $H_{0}$ or its rate of change will appear on the observed signal as an undesirable "field noise."

Thus, the observation of spectra containing very narrow lines by the slow passage technique imposes severe demands on the apparatus as regards stability of operation. For an accurate trace of a spectrum of ethyl alcohol, fluctuations of the field and variation of the sweep rate must be held to less than $10^{-6}$ gauss per second. Furthermore, the fixed transmitter frequency of about $3 \times 10^{7}$ cycles per second cannot be allowed to fluctuate by more than a few hundredths of a cycle per second or about one part in $10^{9}$ during the time in which a resonance is traced.

In addition to the stability requirements outlined above, the study of proton magnetic resonance spectra demands a high degree of homogeneity of the field $H_{0}$ in the region containing the sample. The line spacings encountered in the proton magnetic resonance spectrum of ethyl alcohol are typical of those of organic liquids and range from a fraction of a cycle per second for the finest structures to about one hundred cycles per second for the chemical shifts in a field $H_{0}$ of 7000 gauss. The resolution of a splitting of one-half cycle per second requires that the effective field inhomogeneity over the region of the sample be less than $10^{-4}$ gauss, or about one part in $10^{8}$ of the field used.

\footnotetext{
${ }^{13}$ N. Bloembergen and R. V. Pound, Phys. Rev. 95, 8 (1954).
} 

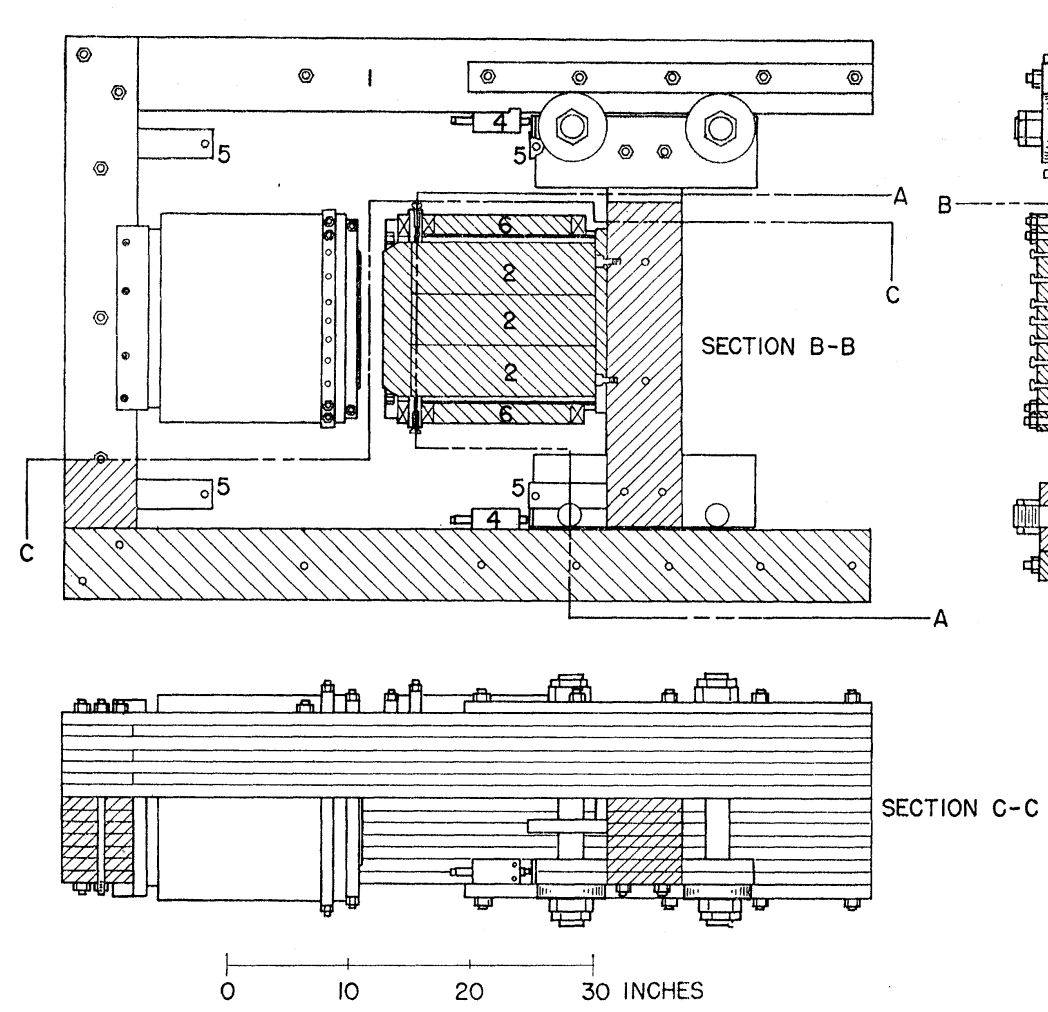

SECTION A-A

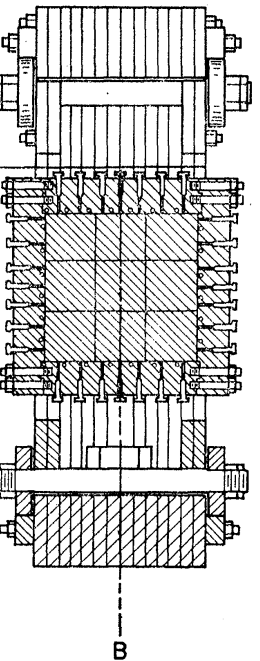

FIG. 1. Detail of permanent magnet. 1Cold-rolled steel yoke; 2-Alnico bars; 3-pole cap; 4-screw jacks; 5 -pads for hydraulic jacks; 6 -energizing and bias coils.
The remainder of this section will describe the development of the apparatus which was guided by the requirements of stability and field homogeneity necessary to the observation of the finest details of the spectra.

\section{a. The Magnet}

The early investigations of proton magnetic resonance spectra were carried out in the field of a carefully shimmed and regulated electromagnet. Although considerable effort was directed toward improving the characteristics of this magnet, the shortcomings, particularly as regards stability of the field, precluded the effective study of the finer details of the proton magnetic resonance spectra. In view of the inherent difficulty of regulation of electromagnets, it was decided that a permanent magnet should be built. The fabrication of this magnet was accomplished in the Stanford University shops. To give a degree of flexibility to its application, the magnet was designed with one hydraulically actuated movable pole so that the length of the gap could be varied. Because of the demand of a high degree of field homogeneity, the magnet was made as large as feasibly possible, and careful attention was directed to its geometry.

Figure 1 shows a plan form and section of the magnet. The 12.6-inch square cross section of the poles $(A A)$ was dictated by the available shapes of Alnico $\mathrm{V}$ blocks. The pole caps are of forged magnetic Armco ingot iron.
As can be seen in section $B-B$, the pole assembly, composed of nine Alnico bars and the pole cap, is held together and cantilevered from the magnet yoke without other fastenings by three frames and twenty-eight prestressed bolts. The framed and bolts are of high tensile strength aluminum alloy, and the frame fastenings are of nonmagnetic stainless steel. Thus, the geometry of the ferromagnetic parts of the pole assembly is very simple.

The alignment of the soft iron yoke was carried out in such a way that the tracks defining the motion of the carriage of the movable pole were accurately perpendicular to the face of the fixed pole, and the carriage wheels were then fabricated to such a size that the movable pole face was aligned parallel to and in register with the fixed pole. Residual misalignment is corrected by a very slight elastic deformation of the yoke through the independent adjustment of four mechanical screwjacks which oppose the large magnetic forces tending to close the gap.

The faces of the Armco pole caps were carefully ground on a grinder having a rotating carriage. This method of grinding provides that any irregularities introduced by the grinder traverse system have axial symmetry. Both front and back faces were ground. In addition, since the lengths of the Alnico bars varied by as much as 0.025 inch, each set of nine bars was clamped together and ground as a unit before the pole caps were set in place. The surfaces of the Alnico bars were not 
entirely free of small flaked pits and voids, but, being separated from the gap by approximately 2 inches of soft iron, these irregularities did not seem to produce noticeable inhomogeneities of the field.

The Alnico bars were assembled in an unmagnetized state and were magnetized after the magnet was completed. A total magnetizing force of about 270000 ampere-turns was furnished by a current pulse of 1500 amperes lasting about one second, distributed over the thirty coils of 180 turns each. After magnetization and the recommended stabilization by a $5 \%$ demagnetization, the gap was opened with the hydraulic jacks, and the field was measured as a function of gap length with a Hall effect fluxmeter. The results are shown in Fig. 2.

At a gap opening of a nominal $1 \frac{1}{2}$ inches, the field intensity has approximately the design value of 7000 gauss, and at this opening the mechanical jacks were installed to take the thrust of the movable pole. After the pole faces had been aligned by these jacks, a preliminary. mapping of the field was carried out. The magnitude of the field has very good circular symmetry as far out as three inches from the center line, and the radial dependence is approximately quadratic. A permanent magnet has fringing fields characteristically larger than those of a comparable electromagnet. In the present magnet, this fringing leads to relatively large radial gradients of the field of the order of 2 gauss per inch at a radius of one inch.

This circularly symmetric inhomogeneity is compensated by a circularly symmetric distribution of small currents on the magnet pole faces. "Current shimming" is accomplished by the independent variation of the current in nine concentric flat spiral coils attached to each pole face. The coils have an average of about fifteen turns, and the currents range from 5 to 45 milliamperes. With proper adjustment of the shim coil currents, largely accomplished by trial and error, the field over a sample $\frac{3}{16}$ inch in diameter can be compensated to an inhomogeneity of less than $\frac{1}{2}$ milligauss in selected regions of the gap. When the field distribution is studied with a highly resolved line, it is apparent that there is a residual inhomogeneity of the field of a lower symmetry which cannot be compensated by the axially symmetric current shims. Part of this variation has a cylindrical symmetry about the horizontal $x$-axis and is probably due to a redistribution of the fringing flux to the yoke and the hydraulic jacks. This distortion amounts to about 0.04 gauss at one inch from the center of the magnet. The remainder of the field inhomogeneity is assumed to come from surface irregularities of the pole faces, and is not larger than may be expected if the pole faces depart from a smooth surface by about $10^{-5}$ inch.

\section{b. Sample Spinning}

Effective inhomogeneity of the field is further reduced by a macroscopic motion imparted to the molecules of

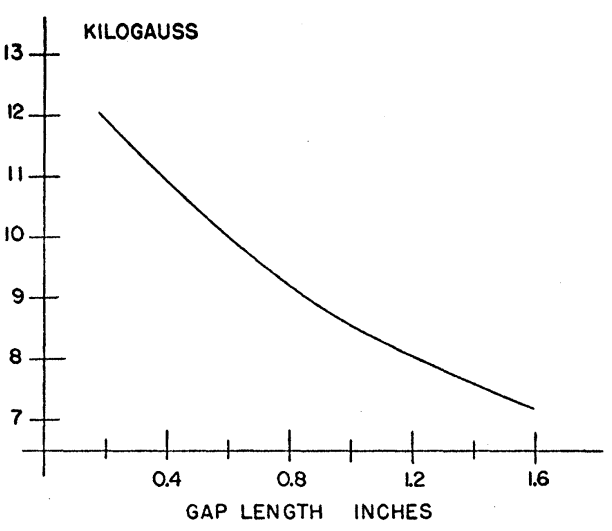

FIG. 2. The field of the permanent magnet as a function of gap length.

the sample. ${ }^{14,15}$ It can be shown that a nucleus exposed to a field which varies in time within limits defined by $\Delta H_{0}$ from an average value exhibits a resonance as though it were exposed to a constant field with a value equal to that of the weighted average of the varying field, provided that the variation has Fourier components only at frequencies which are large compared to $|\gamma| \Delta H_{0}$. If the variation is periodic, small "sideband" resonances will occur on either side of the main resonance at intervals corresponding to the frequency of the periodic variation and its harmonics. However, the side-band resonances will be the smaller, the larger the frequency of the periodic motion.

Similarly, in a field which is constant in time, but has an inhomogeneity defined by $\Delta H_{0}$ over the region of the sample, a nucleus which carries out a periodic macroscopic motion over that region will give rise to a resonance as though it were moving in a constant homogeneous field, so long as the lowest frequency associated with its motion is still large compared to $|\gamma| \Delta H_{0}$. With inhomogeneities, for example, of one milligauss in the region of the sample, the period of the motion must be small compared to about $\frac{1}{5}$ second. This condition may easily be met, at least in the restricted sense of rotation of the sample, with rotational speeds in excess of a few hundred revolutions per minute.

While rotation alone effectively averages only over circles about the axis of rotation, it can be shown as a consequence of Maxwell's equations that if the z-component of the field $H_{0}$ is constant along the axis of rotation, the effective field for the resonances of the sample may be made arbitrarily homogeneous by sufficiently rapid rotation. This fact greatly simplifies the conditions which must be fulfilled in order to observe very high resolution. In a suitably constructed head, the rotation of the sample has allowed the complete resolution of sharp proton magnetic resonance lines separated by as little as one-half cycle per second in $30 \mathrm{Mc} / \mathrm{sec}$.

${ }^{14}$ F. Bloch, Phys. Rev. 94, 496 (1954)

${ }^{15}$ W. A. Anderson and J. T. Arnold, Phys. Rev. 94, 498 (1954). 

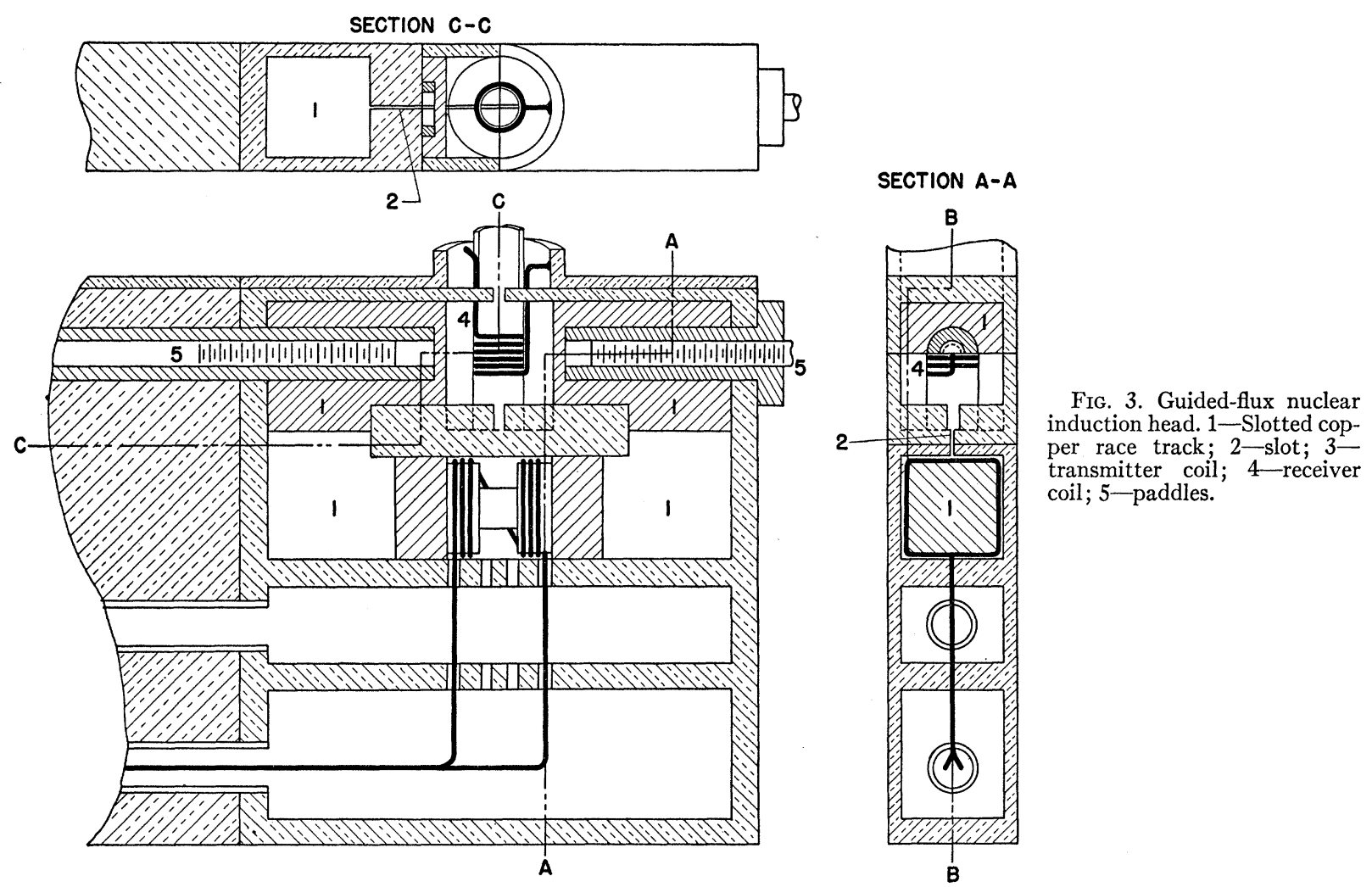

In the apparatus used for high-resolution studies, rotation of the sample was accomplished by attaching the sample holder to a small air turbine with the axis of rotation in the $y$-direction. The rotation must be stable and smooth in order that the microphonic modulation of the radio-frequency coupling be kept within reasonable limits. Several models of rotors were tried; the final one used consists of a turbine attached to a long thin-walled tubular shaft which is supported by teflon bearings. All parts of the turbines and rotors are fabricated from plastics. Rotational speeds up to 10000 revolutions per minute may be realized, but for most of the studies, a few hundred per minute proved adequate, and the lower rotational speeds prolong the life of the bearings.

\section{c. Nuclear Induction Heads}

The first, and incomplete, resolution of the proton magnetic resonance spectrum of ethyl alcohol was observed by using a modification of a head designed by Weaver ${ }^{16}$ in which the transmitter output is led to a pair of transmitter coils of roughly Helmholtz proportions, with the receiver coil and the sample located at the center of symmetry. In principle, when the transmitter and receiver coils are orthogonal, and in the absence of electrostatic coupling, such a head will give an output which represents only the coupling due to the nuclei. In practice, the electrostatic coupling is

\footnotetext{
${ }^{16}$ H. E. Weaver, Jr., Phys. Rev. 89, 923 (1953).
}

difficult to balance even when Faraday shields are interposed between the transmitter and receiver coils, and is subject to microphonic fluctuations.

In view of the difficulty of controlling electrostatic coupling in this configuration, a head was designed and built in which the transmitter coil was located far from the receiver coil, and the radio-frequency flux was guided, by eddy currents induced in what amounts to a slotted copper race track, to the vicinity of the receiver coil and sample. The details of this head are shown in Fig. 3. It is less subject to microphonic noise, and its inherently good geometry makes it easy to balance with small flux steering paddles ${ }^{12}$ when the receiver coil is properly positioned. This head was satisfactory to a large extent, and it was in it that the first sample stirring and rotation experiments were carried out. However, there seemed to be a limit to the improvement of the line width of the resonances possible in this head. It was finally concluded that the geometry of the diamagnetic materials of the head, rotors, and sample holders could introduce gradients of $H_{0}$ which set the lower limit of experimentally obtained line widths, even at selected regions of the magnet gap, to be about two cycles per second. Indeed, with the relatively poor geometry of the parts near the sample, the bulk diamagnetic susceptibilities of from $10^{-6}$ to $10^{-5}$ could introduce gradients of the $z$-component of $H_{0}$ of as much as 0.1 gauss per centimeter in the sample region with fields of 7000 gauss. A most fortuitous matching 
of this inhomogeneity by compensating gradients due to shim currents or to the magnet itself could not be expected to reduce it by factors of more than 100 .

With this consideration in view, a head was designed and built in which the dominant geometry has cylindrical symmetry about the $y$-direction, taking advantage of the fact that a long cylinder of finite susceptibility in a uniform field has within itself a uniform field, except for end effects. The ends of the various cylinders which make up the head are sufficiently removed from the center at which the receiver coil and spherical sample are located that the effects of the diamagnetic susceptibility lead only to very small gradients of $H_{0}$ along the $y$-direction. Figure 4 shows a sectional view of this head design, and of the sample holder used. The latter is made in such a way that when assembled it becomes a homogeneous cylinder with a centrally located spherical cavity for the sample. Here, advantage is taken of the fact that a sphere, with a susceptibility differing from that of its surrounding medium, in a homogeneous field has within itself a homogeneous field. It will be noted that the metal parts which perforce cannot occupy perfect cylindrical symmetry are none the less symmetrically placed about the $y-z$ and $x-z$ planes. Fortunately, the bulk susceptibilities of copper and the plastics used (Rexolite and Lucite), are very nearly the same. The copper parts, moreover, are made as thin as possible, and are recessed in the plastic parts so as to leave no air spaces. The ribbon constituting the receiver coil and its leads, for example, has a thickness of 0.003 inch and rests in shallow grooves in the plastic supports.

While it is true that the presence of the copper shields and wires increases somewhat the radial and azimuthal gradients of $H_{0}$ in the $x-z$ planes, the rotation of the sample about the $y$-direction is very effective in averaging in these planes, and it remains only to adjust the current shims and magnet geometry to minimize the variation of $H_{0}$ in the $y$-direction.

It should be pointed out that in the case of all heads used in high-resolution nuclear magnetic resonance studies, the materials had to be selected carefully for freedom from ferromagnetic inclusions. Commercial brass is usually poor in this respect, and in the heads constructed for the highest resolution, the metal used was therefore in most cases electrolytic copper. Most commercial plastics tried, for example Lucite, Rexolite, and polystyrene, seem to be uniformly free of ferromagnetic contamination. Teflon is an exception in that it sometimes shows contamination. After fabrication, the parts had to be carefully cleaned of surface contamination by iron particles from tools used, and even from dust which is laden with ferromagnetic particles, especially in shop spaces. Considerable reliance could be placed in soaking the parts in hydrochloric acid which dissolves any surface deposits of iron.

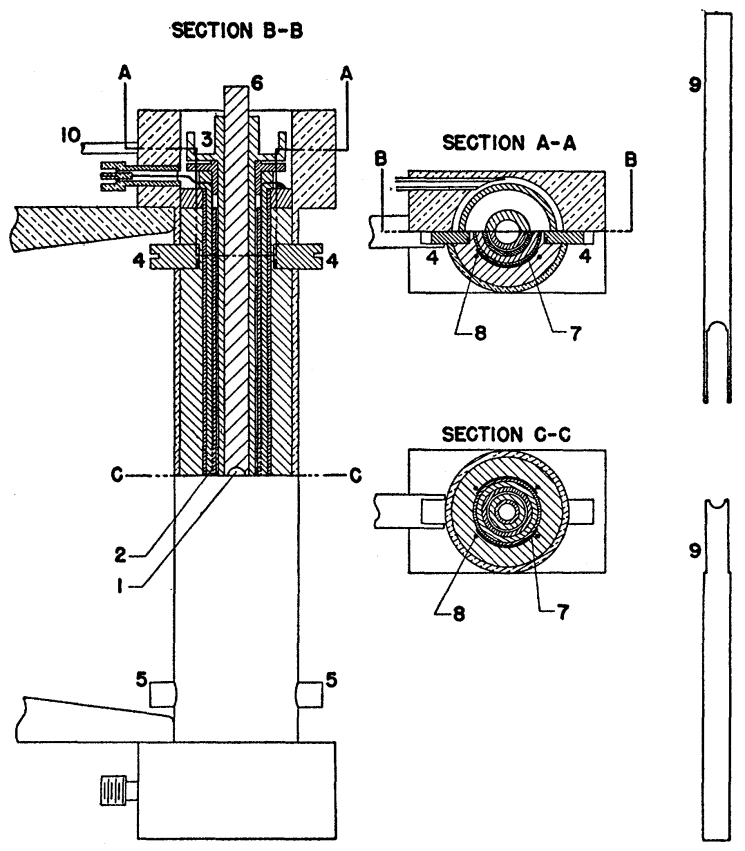

FIG. 4. High-resolution nuclear induction head. 1-Sample; 2-receiver coil; 3-air turbine rotor; 4-paddles; 5-dummy paddles; 6-sample holder; 7-copper flux guiding shield; 8transmitter wire; 9 -detail of sample holder; 10-air-jet inlet.

\section{d. Radio-Frequency System}

Figure 5 represents the final configuration of the radio-frequency system in a block diagram. The particular arrangement was developed to meet conveniently the requirements imposed by the extreme narrowness and low signal strength of the resonances studied, although the exact configuration is somewhat arbitrary.

\section{Transmitter}

It was pointed out above that for effective study of the narrowest resonances of protons in liquids, the transmitter must have a frequency stability approaching one part in $10^{\circ}$. In this respect, an ordinary quartzcrystal-controlled oscillator is not satisfactory. After some unsuccessful trials with crystal-controlled oscillators of conventional design, operated from dry-battery supplies, a stable oscillator of the Meacham crystal bridge type ${ }^{17}$ was built and used. The output frequency of the oscillator is multiplied in the first stage of an amplifier unit. The final stage of the amplifier is neutralized so that the output to the head can be reduced to a very low level by a simple adjustment of grid bias. The entire amplifier unit is rigidly attached to the head to minimize cable microphonic and phase shift noise.

\section{Receiver}

Since the radio-frequency voltages induced by the proton magnetic resonances are very small, the first

\footnotetext{
${ }^{17}$ L. A. Meacham, Proc. Inst. Radio Engrs. 26, 1278 (1938).
} 


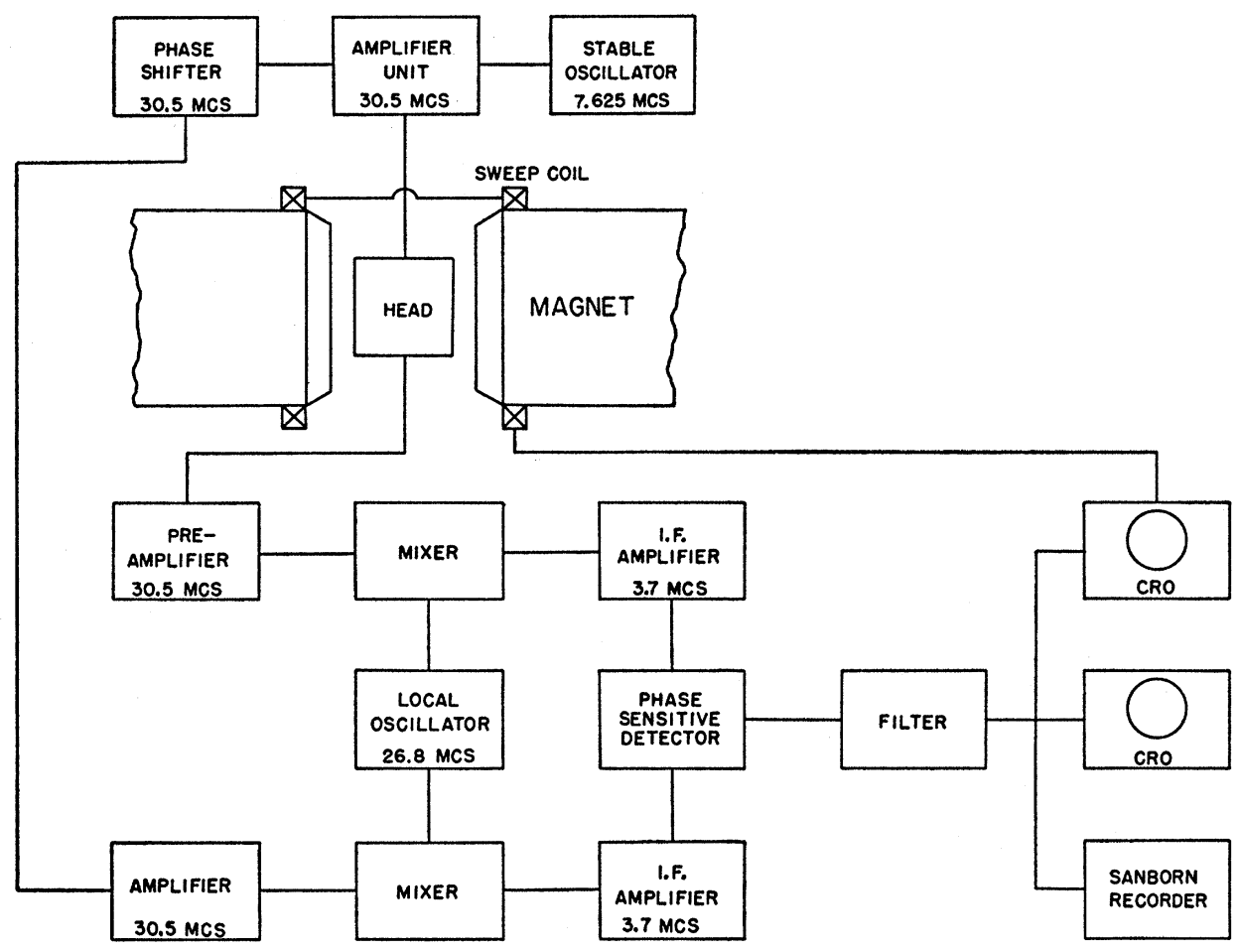

FIg. 5. Radio-frequency and display system. requirement of the receiver is that it introduce as little noise as possible. The preamplifier at 30.5 megacycles is of the Wallman cascode design,${ }^{18}$ having two complete stages and a voltage gain of approximately 100 . This amplifier contributes most of the noise in the receiver, and the over-all noise voltage which the receiver system introduces is typically about four times the Johnson noise generated in the shunt resistance of the resonated receiver coil of the head. The second requirement of the receiver is that it should have a voltage gain large enough to drive the various display units. The superheterodyne configuration is convenient, since it offers high gain without the instability of a single frequency amplifier and without the tendency to drift found in high gain direct-current amplifiers. The intermediate frequency amplifier has a single high gain stage at about 3.7 megacycles. The band width of the receiver is primarily reduced by filtering out high frequencies after second detection, although the interstage couplings in the radio-frequency and intermediate-frequency sections, with $Q$-values of about 50, also help to limit the pass band. The final band width is reduced to about one cycle per second for the slowest traces.

\section{Mode Discrimination}

Although the heads used are provided with flux steering paddles ${ }^{12}$ for balance of in-phase and quadrature coupling, these are used primarily only to reduce the coupling between the transmitter and receiver to

${ }^{18}$ Wallman, Macnee, and Gadsden, Proc. Inst. Radio Engrs. 36, 700 (1948). a point low enough to prevent saturation of the amplifiers. For the selection of the desired mode, a phase sensitive detector is used. The reference voltage necessary to the operation of this detector is derived from the transmitter and passes through an auxiliary superheterodyne receiver using the same local oscillator as the main receiver. Phase shifts are preserved in the frequency conversion of both receivers, and the phase sensitive detector rejects the component of the receiver signal which is in quadrature to the reference signal. The reference signal amplifier is provided with a variable phase shifter, and with this control, signals of a welldefined phase may be selected. In particular, signals representing very pure absorption mode may be obtained with this type of detector.

The output of the detector drives a cathode follower which isolates it from the display units. The signals are displayed on long persistence oscilloscopes, and, when desired, on paper tape with a Sanborn recorder.

\section{e. Field and Sweep Control}

Changes in temperature of the permanent magnet produce changes in the field which, near room temperature, amount to about one part in 5000 per degree centigrade. With its very large heat capacity, the magnet responds very slowly to changes in ambient temperature. Nevertheless, if the temperature of the room differs by as little as one or two degrees from that of the magnet, the temperature-dependent drift of the magnet becomes comparable to or greater than the desired sweep rates. This is not unreasonable in view of 
the fact that the thermal relaxation time of the magnet is of the order of six hours, and a two-degree difference between the magnet and the ambient air leads to a temperature drift of about $10^{-4}$ gauss per second which is ten times the lowest desired sweep rate.

To counteract temperature drifts and to control the field in such a way that resonances may be observed at a fixed frequency in spite of temperature changes, a small adjustable bias field is provided by small currents in the magnetizing coils to change $H_{0}$ by amounts up to about $0.1 \%$. This bias field, always less than ten gauss, is superposed on that of the magnet so as to increase $H_{0}$, and there is no measurable irreversibility in its effect on the magnet. The temperature of the room is controlled as well as conveniently possible. Residual temperature-dependent drifts of the field are approximately compensated by causing the bias current to change automatically at a small and constant rate which must be adjusted from time to time.

The current supplies to both the bias and shim coils must of necessity come from very stable sources. For example, a fluctuation of the bias current of one microampere changes the field by about $10^{-4}$ gauss, which is comparable to the line widths of the resonances. Storage batteries of low internal resistance were used in circuits designed to keep their net current equal to zero. Even with the batteries functioning as very effective filters and regulators, careful attention has to be directed to the regulation and filtering of their charging sources.

The necessary sweep fields are produced in auxiliary bias coils by currents which simultaneously energize the deflecting coils of a magnetic deflection cathode ray oscilloscope. The currents are derived from a sawtooth signal generator of the "bootstrap" variety, whose output is linear with time to about one percent. Repetition rates from 0.001 per second to 10 per second are available. The extreme limits of the sweep field are controlled by a variable resistance shunted across the sweep coils of the magnet. This control provides for sweeps of from $10^{-3}$ gauss to 0.5 gauss.

\section{MEASUREMENTS}

Although final measurements were made in slow passage with an unmodulated transmitter field of fixed frequency and with $H_{0}$ constant except for the slow linear sweep, the transient behavior ${ }^{19}$ of the resonances under conditions of rapid passage and the side-band presentation resulting from the modulation of either the transmitter field or $H_{0}$ were often used to establish the conditions under which the final traces were made.

The transient behavior of the resonances has been of great importance also in the prediction of structure in the resonances observed. A line will display under rapid passage a transient oscillatory signal whose period depends on the sweep rate and decreases as the sweep

${ }^{19}$ B. A. Jacobson and R. K. Wangsness, Phys. Rev. 73, 942 (1948). progresses. The envelope of this oscillatory transient is characteristic of the true line shape and of the field inhomogeneity over the region of the sample. For example, in a homogeneous field, if the resonance line is single, the transient signal will decay in a true exponential fashion with a time constant equal to the transverse relaxation time. In an inhomogeneous field the decay will in general be quicker and will be a superposition of exponentials. If the line is a multiplet the decay will display a modulation due to constructive interference of the beats of various components of the multiplet. It was the observation of modulated transients which indicated that the resonances encountered in ethyl alcohol could be further resolved, and thus stimulated the efforts to achieve higher resolution. Also, observation of the transient behavior of the signals is useful in the adjustment of the magnet for the best resolution.

A good many precautions must be undertaken to achieve the highest stability and resolution in the apparatus. The stability of the field is influenced both by temperature fluctuations and by unshielded fluctuating fields coming from other magnets in the laboratory, and even from disturbances of the magnet fringing field due to such items as pocket knives carried by laboratory personnel. Furthermore, some power supplies, although electronically regulated, were connected to the power mains whose voltage fluctuates when large changes in demand occur. For these reasons, the apparatus was usually operated late at night to obtain the very slow traces of the spectra.

The procedures which are used to obtain the highest resolution follow several steps. First, to facilitate adjustment of the geometry of the magnet and the current shims, resonances of the single line in water are observed. Best results are obtained if the field is first made approximately uniform over a relatively large region at the center of the magnet. The ultimate resolution is then found by searching in the field for the best spot, using the transient decay of a rapid passage signal as an indicator of field homogeneity. In general, the longer the transient signals persist, the more homogeneous the field in the region of the sample.

In the preparation of a sample of ethyl alcohol for the highest resolution traces, several precautions had to be observed. Sample holders had to be filled in such a way as not to permit inclusion of any gas bubbles which would destroy the spherical symmetry of the sample. Samples also had to be prepared and stored under an inert atmosphere such as nitrogen. Ethyl alcohol which has been exposed to air appears to display resonance lines which are so broad as to obscure the finest details of the spectra. This effect is not completely understood, although it is possible that the dissolved oxygen of the air provides a paramagnetic relaxation. If dissolved oxygen is the line broadening agent, it 
must be considerably more influential in the case of ethyl alcohol than in the case of water..$^{20}$

The precaution in the preparation of samples under an inert atmosphere was first undertaken in the investigation of the effects of very small concentrations of acid or base in ethyl alcohol (see Sec. IV.d), in order to keep the concentration stable. The enhanced resolution was found as a fortuitous by-product.

The frequency intervals between the lines of the spectra were calibrated by side-band lines of a resonance, which occur when either the transmitter field or $H_{0}$ is modulated. If the modulation frequency is $\omega^{\prime}$, sideband lines appear at intervals of $\omega^{\prime}$ from the central resonance. This useful calibration method obviates the necessity for very accurate calibration of the transmitter frequency in finding sweep rates and line intervals in the spectra.

\section{RESULTS}

This study of a highly resolved proton magnetic resonance spectrum of ethyl alcohol deals with two distinct molecular phenomena. The first concerns the frequency intervals and intensities of the resonances and the incorporation of these quantities in the energy level scheme of a simple Hamiltonian, to be presented in Secs. a, b, and c below. The second involves the possibility of exchange of protons between molecules and will be discussed in Sec. d. Actually, the study of each of these phenomena has led to discoveries regarding the other.

\section{a. Chemical Shifts}

The first degree of resolution of the proton magnetic resonance spectrum of ethyl alcohol is shown in Fig. 6(a), and has already been reported. ${ }^{8}$ Using the accepted chemical terminology for the $\mathrm{CH}_{3}$, the $\mathrm{CH}_{2}$,

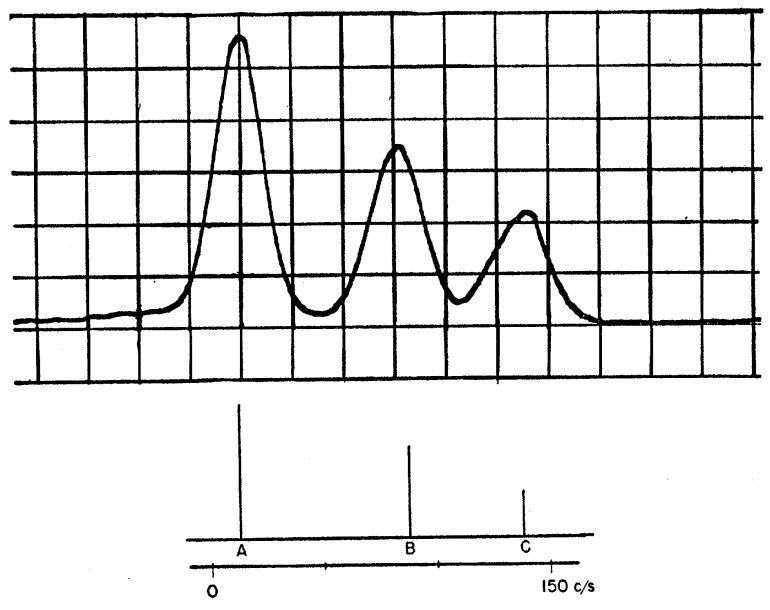

(a)

FIG. 6(a). Spectrum of ethyl alcohol sufficiently resolved to show the internal chemical shift.

${ }^{20}$ A. Chiarotti and L. Guilotto, Phys. Rev. 93, 1241 (1954). and the $\mathrm{OH}$ groups of protons, the three lines $A, B$, and $C$ were assigned to the methyl, the methylene, and the hydroxyl groups respectively. At this stage of resolution, the displacement of the lines from each other is due to the internal chemical shifts. They have previously been found to be proportional to the external field ${ }^{6}$ and are attributed to an effective shielding of the nuclei by the electrons of the molecule. The shielding is greatest for the methyl group, becoming progressively less for the methylene and hydroxyl groups; that is to say, in a given applied field the methyl group protons have their resonance at the lowest frequency and the resonances of the methylene and hydroxyl groups follow in sequence. In the experiments it was actually the field $H_{0}$ rather than the frequency which was varied.

Chemical shifts may be described in the Hamiltonian representing the spin energy of the entire molecule by adding to the external field $H_{0}$ an additional effective molecular field $H_{K}$ which, for a given $H_{0}$, has different values for the different groups of protons. The protons in a particular chemical group $K$ are assumed to be equivalent, and their spins add to give a total spin angular momentum $I_{K}$ which is a constant of motion as a consequence of the assumed form of the Hamiltonian. If $n$ is the number of protons in the group $K$, $I_{K}^{2}>0$ takes the values $\left(\frac{1}{2} n+1\right)\left(\frac{1}{2} n\right),\left(\frac{1}{2} n\right)\left(\frac{1}{2} n-1\right)$, $\left(\frac{1}{2} n-1\right)\left(\frac{1}{2} n-2\right)$, etc. Disregarding the possible interactions between protons in different chemical groups, the Hamiltonian has the form

$$
\begin{aligned}
\mathfrak{F}_{1}=-\hbar|\gamma|\left\{\left(\mathbf{H}_{0}+\mathbf{H}_{A}\right) \cdot \mathbf{I}_{A}+\left(\mathbf{H}_{0}+\mathbf{H}_{B}\right) \cdot \mathbf{I}_{B}\right. \\
\\
\left.+\left(\mathbf{H}_{0}+\mathbf{H}_{C}\right) \cdot \mathbf{I}_{C}\right\},
\end{aligned}
$$

with $A, B$, and $C$ referring to the three groups of protons
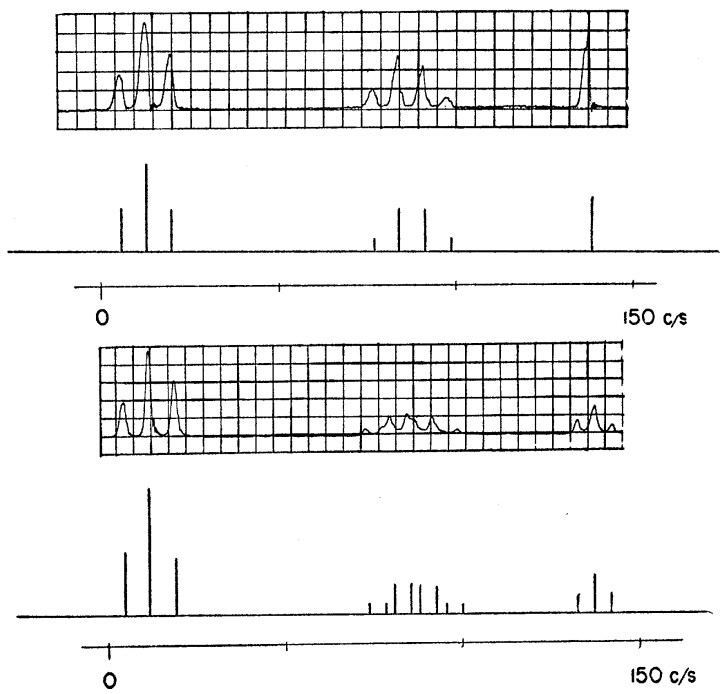

(b)

FIG. 6(b). Spectra of ethyl alcohol in the second degree of resolution. The upper trace originates from impure ethyl alcohol; the lower, from pure ethyl alcohol. 

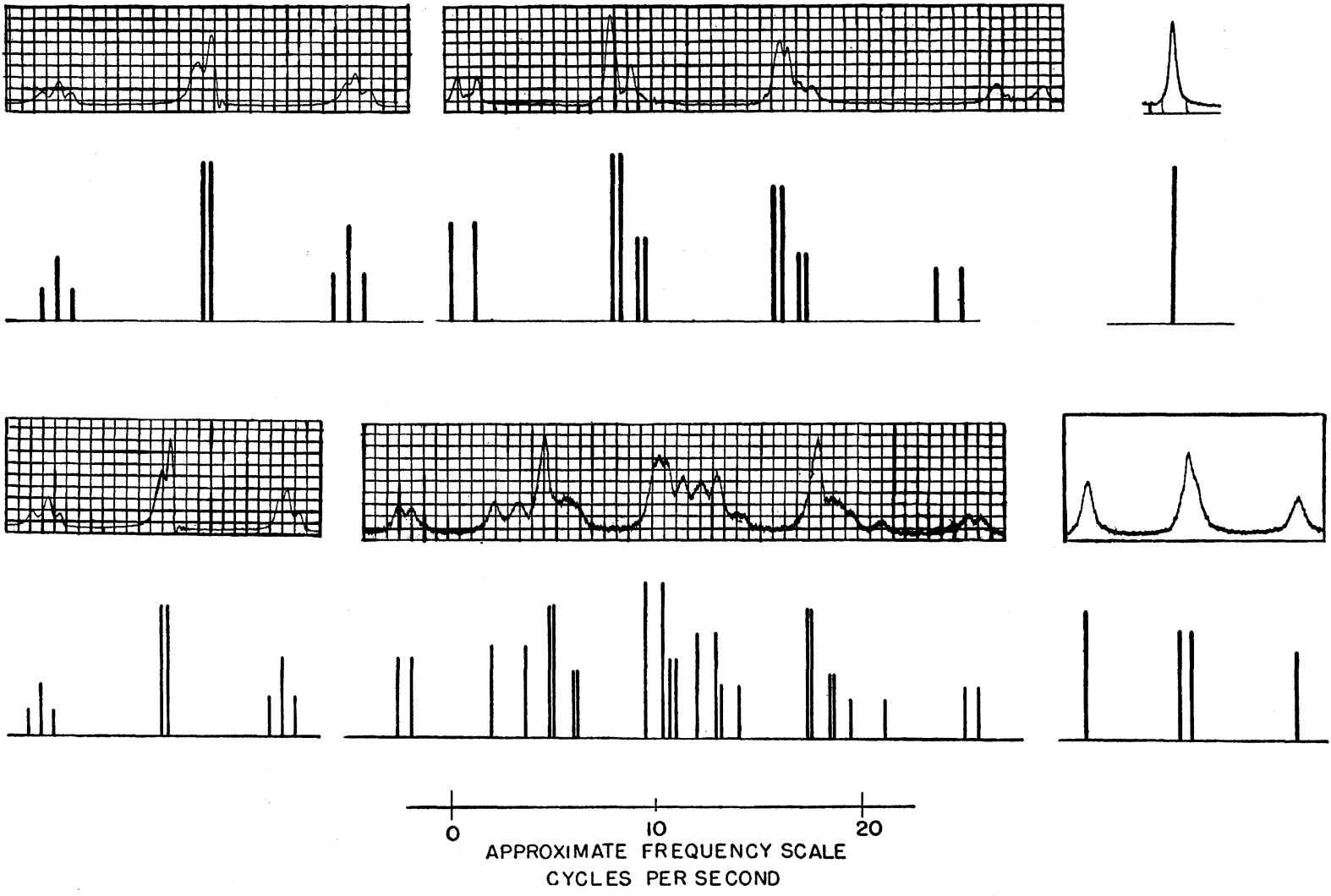

(c)

FIG. 6(c). High resolution traces of the methyl, methylene, and hydroxyl groups in sequence from left to right. The three top traces originate from impure ethyl alcohol; those below, from pure ethyl alcohol.

which were introduced above. The expressions for the energy levels of (1), characterized by the eigenvalues $m_{A}, m_{B}$, and $m_{C}$ of $I_{z A}, I_{z B}$, and $I_{z} C$, respectively, are given by

$$
\begin{aligned}
E_{m_{A} m_{B} m_{C}}{ }^{(0)}=-\hbar\left\{\left(\omega_{0}+\omega_{A}\right) m_{A}+\right. & \left(\omega_{0}+\omega_{B}\right) m_{B} \\
& \left.+\left(\omega_{0}+\omega_{C}\right) m_{C}\right\}
\end{aligned}
$$

where $\omega_{0}=|\gamma| H_{0}$ and $\omega_{K}=|\gamma| H_{K}$. The energy level scheme (2) gives the three chemically shifted lines drawn in Fig. 6(a) below the experimental trace when transitions of $\Delta m= \pm 1$ are allowed for one group at a time. The placement of the zero on this scale has no particular meaning. The intensities of the three lines indicated by the height of the lines in the diagram, and defined by the areas under the peaks of the experimental trace must be expected to be in the ratio $3: 2: 1$, corresponding to the number of protons in the groups $A, B$, and $C$. This intensity ratio is verified in the trace to within the limits of experimental accuracy.

\section{b. First Order Spin-Spin Interaction}

Figure 6(b) shows the traces of the ethyl alcohol spectrum under higher resolution. Their form is dependent on the purity of the sample used, and the two extreme cases presented here represent "impure" and "pure" ethyl alcohol. These designations refer to the sufficient presence or almost complete absence of contaminating $\mathrm{H}^{+}$or $\mathrm{OH}^{-}$ions, respectively. It will be noted that in these traces the chemically shifted lines, resolved in Fig. 6(a) have structure. The number and intensities of the lines in each group suggest that the splitting of the resonances of one group of chemically equivalent protons depends on the states of the protons in adjacent groups. There is much evidence to support this view. A technique of confirming it directly in the case of ethyl alcohol uses a method of simultaneous irradiation of pairs of interacting groups..$^{21-23}$

The application of this technique is presented by Anderson $^{10}$ in the following paper. It is particularly useful in the cases of molecules in which the resonances of the interacting groups are less easily identified.

In the simpler case of impure ethyl alcohol, the only noticeable interaction between protons in the molecule is that taking place between the methyl and methylene groups. The methyl group resonances constitute a triplet, and those in the methylene group a quartet of lines. The fact that the hydroxyl group contributes a single line is interpreted to indicate that its interaction

${ }^{21}$ F. Bloch, reference 9; see Sec. 7.
${ }^{22}$ F. Bloch, Phys. Rev. 93, 944 (1954).

${ }^{23}$ A. L. Bloom and J. N. Schoolery, Phys. Rev. 97, 1261 (1955). 
with the other proton groups is in this case averaged out by rapid exchange of hydroxyl protons between different molecules (see Sec. d). The structures of the methyl and methylene groups have the same frequency intervals $J_{A B}$. Except for the ambiguity of the sign of $J_{A B}$, it is possible to assign to each component of one group of lines the respective magnetic quantum number of the neighbor group.

In this degree of resolution, the line spacings may be understood from a first-order calculation of a more complete Hamiltonian than (1) which has, in addition to the terms of (1), a term which describes the interaction between the protons of the methyl and methylene groups $^{6,7}$ :

$$
\mathfrak{H}_{2}=\mathcal{H}_{1}-\hbar J_{A B} \mathbf{I}_{A} \cdot \mathbf{I}_{B}
$$

A first-order expression for the energy levels of (3) may be found by computing the diagonal elements of $\mathcal{H C}_{2}$ in the $I_{z}$ representation. They are obtained simply by replacing the operators $I_{A}, I_{B}$, and $I_{C}$, by the respective eigenvalues $m_{A}, m_{B}$, and $m_{C}$, of $I_{z A}, I_{z B}$, and $I_{z C}$, respectively; thus

$$
\begin{aligned}
E m_{A} m_{B} m_{C}(1)=-\hbar\left\{\left(\omega_{0}\right.\right. & \left.+\omega_{A}\right) m_{A}+\left(\omega_{0}+\omega_{B}\right) m_{B} \\
& \left.+\left(\omega_{0}+\omega_{C}\right) m_{C}+J_{A B} m_{A} m_{B}\right\} .
\end{aligned}
$$

Transitions for which $m$ changes by \pm 1 on one group at a time give rise to the various resonances. Thus, the three frequencies in group $A$ are given from (4) by

$$
\omega_{0}+\omega_{A}+J_{A B} m_{B}
$$

with $m_{B}$ taking the values of 1,0 , and -1 . Their intensities can in this approximation be expected to be in the ratio of $1: 2: 1$ corresponding to the binomial coefficients of two; that is, corresponding to the number of ways in which each of the values of $m_{B}$ can be found by combining the two spins of $\frac{1}{2}$ of the protons in group $B$. Similarly, the four frequencies in group $B$ are given by

$$
\omega_{0}+\omega_{B}+J_{A B} m_{A},
$$

with $m_{A}$ taking the values $\frac{3}{2}, \frac{1}{2},-\frac{1}{2},-\frac{3}{2}$, and the intensity ratios expected in this approximation are $1: 3: 3: 1$, the binomial coefficients of three.

The slightly more complicated case of pure ethyl alcohol may be described by a superposition in group $B$ of the splitting of the interacting groups. In this case, the interaction between the methylene and hydroxyl groups of protons is not completely averaged out by exchange of protons of the latter group. To describe this interaction, one more term must be added to the Hamiltonian (1); thus for pure ethyl alcohol

$$
\mathcal{H}_{2}=\mathcal{H}_{1}-\hbar\left\{J_{A B} \mathbf{I}_{A} \cdot \mathbf{I}_{B}+J_{B C} \mathbf{I}_{B} \cdot \mathbf{I}_{C}\right\} \text {. }
$$

There is no evidence of an interaction between the methyl and hydroxyl groups, presumably because it is too small to be seen as a consequence of the larger distance in the molecule between the protons corresponding to these groups. In analogy to (4), the energy levels of (5) are found in first order to be

$$
\begin{aligned}
& E m_{A} m_{B} m_{C}{ }^{(1)}=-\hbar\left\{\left(\omega_{0}+\omega_{A}\right) m_{A}+\left(\omega_{0}+\omega_{B}\right) m_{B}\right. \\
& \left.+\left(\omega_{0}+\omega_{C}\right) m_{C}+J_{A B} m_{A} m_{B}+J_{B C} m_{B} m_{C}\right\} .
\end{aligned}
$$

The frequency intervals for group $A$ will be the same as in the case of impure ethyl alcohol, as will be the predicted intensity ratios. The splitting in group $B$ due to the interaction with the protons in group $C$ can be expected in this approximation to amount simply to a doubling of the lines already split by the interaction between the protons of groups $A$ and $B$. The frequency interval of this doubling will be $J_{B C}$. In this approximation, the intensities of the doubled lines, corresponding to the two equally weighted values of $m_{C}$, should within each pair be the same. Thus the total display of the methylene group should have eight lines, with each of the four components which appeared in the case of impure ethyl alcohol now being doubled by the influence of the hydroxyl proton. Experimentally it is found that $J_{A B}>J_{B C}$, hence the arrangement of the lines for the methylene group in pure alcohol should to first approximation show the intensity ratios

$$
\frac{1}{2}: \frac{1}{2}: \frac{3}{2}: \frac{3}{2}: \frac{3}{2}: \frac{3}{2}: \frac{1}{2}: \frac{1}{2} \text {. }
$$

The hydroxyl group in this approximation should show a triplet structure due to the interaction with protons in the methylene group analogous to the triplet structure of the methyl group described above. The interval between the components of this triplet is $J_{B C}$, and the intensity ratio $1: 2: 1$.

In Fig. 6(b), the diagrams below the experimental traces show the spectra predicted from allowed transitions between the energy levels given in (4) and (6). The heights of the lines in the diagrams indicate the intensities predicted in this approximation and should be compared with the areas under the corresponding lines in the traces. The discrepancies are obvious, though relatively small. They are attributable to higher order effects. In particular the evident asymmetry in the components of the methyl and methylene resonances can be explained to within the experimental error by a correction of the intensity ratios, cited above, which is of the order of the ratio of the spin-spin splitting to the difference of the chemical shifts. ${ }^{10}$

From the experimental traces in this degree of resolution it is possible to evaluate the four parameters, $\left(\omega_{B}-\omega_{A}\right),\left(\omega_{C}-\omega_{B}\right),\left|J_{A B}\right|$, and $\left|J_{B C}\right|$, which characterize the spectrum of ethyl alcohol. The experimental values are given in Table $\mathrm{I}$.

\section{c. Spin-Spin Interaction in Higher Order}

The traces of the ethyl alcohol spectrum shown in Fig. 6(c) have nearly the highest resolution so far 
achieved. They do represent the best over-all conditions when the factors of stability of the field and transmitter frequency as well as the homogeneity of the field are considered. It is seen from the traces that the apparently single lines of Fig. $6(\mathrm{~b})$ consist actually each of several components. For example, the two lines of the methyl group corresponding to $m_{B}= \pm 1$ consist each of three components. The explanation of this fact can be found by calculating the eigenvalues of $\mathfrak{F}_{2}$ in Eq. (3) in the second order with the quantity $J_{A B} /\left(\omega_{A}-\omega_{B}\right)$ as the perturbation parameter. In this calculation, there appear terms which are proportional to $J_{A B}{ }^{2}$ and are quadratic in $m_{A}$ with the result that the transition frequencies for $m_{A}= \pm 1$, found from the differences of the energy levels, depend on the initial (or final) value of $m_{A}$, and have different values for the three transitions: $m_{A}: \frac{3}{2} \rightarrow \frac{1}{2}, \frac{1}{2} \rightarrow-\frac{1}{2},-\frac{1}{2} \rightarrow-\frac{3}{2}$.

These terms are absent in the case of the line of group $A$ corresponding to $m_{B}=0$, and in this case one would expect to find a single line. The actual observation of two lines here is explained by the fact that $m_{B}=0$ occurs both for the case of the total spin $I_{B}=1$ and the case of $I_{B}=0$, and that the energy levels depend not only upon $m_{B}$ but also upon $I_{B}$.

It is interesting to note that the line in group $A$ corresponding to the singlet state, $I_{B}=0$, of the methylene group, is considerably sharper than those corresponding to $I_{B}=1$. A qualitative explanation of this fact can be found in the circumstance that the width of the lines in group $A$ is largely determined by the lifetimes of the states pertaining to group $B,{ }^{9}$ and that a change of $m_{B}$ from 0 to \pm 1 involves, for the initial value $I_{B}=0$, a relatively improbable transition from a singlet to a triplet state, whereas for the initial value of $I_{B}=1$, changes of $m_{B}$ simply involve transitions within the triplet state.

A similar effect was sought in the resonances of the hydroxyl group of pure ethyl alcohol, but even in the purest ethyl alcohol obtainable, this effect is almost obscured, probably by exchange broadening. There is evidence that the single sharp line of the hydroxyl group in impure ethyl alcohol occurs very nearly at the position at which the "singlet" line would be expected to occur in the broadened central peak of the hydroxyl group of lines in a pure sample.

TABLE I. Measured values for chemical shift and spin-spin interaction for ethyl alcohol.

\begin{tabular}{ccc}
\hline \hline $\begin{array}{c}\text { Relative chemical } \\
\text { shift at } 30.5 \mathrm{Mc} / \mathrm{sec}\end{array}$ & Spin-spin interaction & $\begin{array}{c}\text { Frequency in } \\
\text { cycles/sec }\end{array}$ \\
\hline$\left(\omega_{B}-\omega_{A}\right) / 2 \pi$ & & $75.0 \pm 1$ \\
$\left(\omega_{C}-\omega_{B}\right) / 2 \pi$ & & $50.5 \pm 1^{\mathrm{a}}$ \\
& $\left|J_{A B}\right| / 2 \pi$ & $7.15 \pm 0.25$ \\
& $\left|J_{B C}\right| / 2 \pi$ & $4.8 \pm 0.2$ \\
\hline
\end{tabular}

a. $27^{\circ} \mathrm{C}$.
Up to terms of the second order, the energy levels derived from (3) are calculated ${ }^{8}$ to be

$$
\begin{aligned}
& E_{I_{A} m_{A} I_{B} m_{B} I_{C} m_{C}{ }^{(2)}=-\hbar\left\{\left(\omega_{0}+\omega_{A}\right) m_{A}\right.} \\
& +\left(\omega_{0}+\omega_{B}\right) m_{B}+\left(\omega_{0}+\omega_{C}\right) m_{C}+J_{A B} m_{A} m_{B} \\
& -\frac{J_{A B}{ }^{2}}{2\left(\omega_{B}-\omega_{A}\right)}\left[m_{A}\left(I_{B}{ }^{2}+I_{B}-m_{B}{ }^{2}\right)\right. \\
& \left.\left.-m_{B}\left(I_{A}{ }^{2}+I_{A}-m_{A}{ }^{2}\right)\right]\right\}
\end{aligned}
$$

for impure ethyl alcohol. Similarly, for pure ethyl alcohol, the energy levels arising from (5) are

$$
\begin{aligned}
& E_{I_{A} m_{A} I_{B} m_{B} I_{C} m_{C}{ }^{(2)}=-\hbar\left\{\left(\omega_{0}+\omega_{A}\right) m_{A}\right.} \\
& +\left(\omega_{0}+\omega_{B}\right) m_{B}+\left(\omega_{0}+\omega_{C}\right) m_{C}+J_{A B} m_{A} m_{B} \\
& -\frac{J_{A B}{ }^{2}}{2\left(\omega_{B}-\omega_{A}\right)}\left[m_{A}\left(I_{B}^{2}+I_{B}-m_{B}^{2}\right)\right. \\
& \left.-m_{B}\left(I_{A}^{2}+I_{A}-m_{A}^{2}\right)\right]+J_{B C} m_{B} m_{C} \\
& -\frac{J_{B C} C^{2}}{2\left(\omega_{C}-\omega_{B}\right)}\left[m_{B}\left(I_{C}^{2}+I_{C}-m_{C}^{2}\right)\right. \\
& \left.\left.-m_{C}\left(I_{B}^{2}+I_{B}-m_{B}^{2}\right)\right]\right\} .
\end{aligned}
$$

The calculation of the ratios of the intensity maxima in higher order is far more involved and cannot, in fact, be rigorously given without an accompanying theory of line widths which must be expected to be different for different lines of the spectrum. It can be shown, however, ${ }^{24}$ that the integrated intensity of each resonance line is proportional to the square of the matrix element of $I_{x}+i I_{y}$, where $I_{x}$ and $I_{y}$ are the $x$ - and $y$-components of the spin vector pertaining to the nucleus in question. To the extent to which equal line widths may be assumed, this quantity gives also a measure for the corresponding intensity maximum. Explicit calculations of the matrix elements have been carried out by Anderson. ${ }^{10}$ The intensities resulting from his calculations are represented by the heights of the lines appearing in the diagrams below the experimental traces in Fig. 6(c).

It will be noted that the frequencies of the resonances in the experimental traces do not coincide exactly with those shown in the diagrams, even when allowances are made for nonuniform sweep rates. A third order perturbation calculation of the energy levels ${ }^{10}$ leads to

\footnotetext{
${ }^{24}$ F. Bloch, reference 9; see Sec. V.
} 

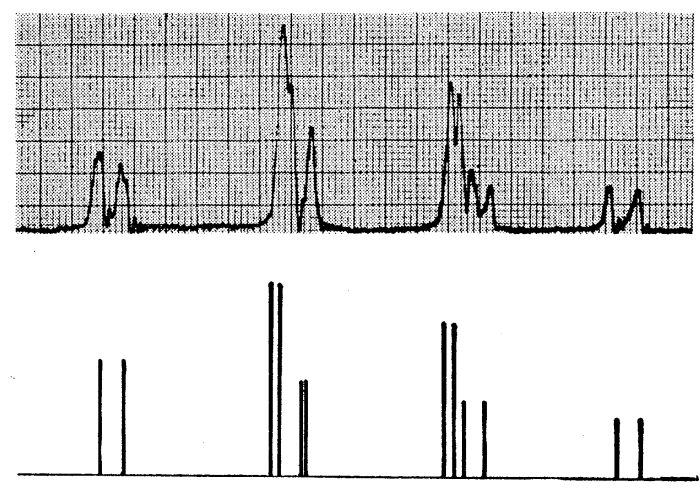

Fig. 7. High-resolution trace of the methylene group originating from impure ethyl alcohol with a diagram of the spectrum as calculated to the third order.

better agreement which is illustrated for the methylene group of impure ethyl alcohol in Fig. 7. It will be noted that the frequencies calculated in third order agree remarkably well with those of the experimental trace.

\section{d. Effects of Contamination with $\mathrm{H}^{+}$and $\mathrm{OH}^{-}$Ions}

It appeared originally ${ }^{25}$ that the resonance of the hydroxyl protons of ethyl alcohol was a single line even though the other chemically shifted groups were split. This lack of structure is now understood to be the effect of a slight contamination with an excess of $\mathrm{H}^{+}$ or $\mathrm{OH}^{-}$ions. The contamination necessary to obliterate completely the structure of the resonance of the hydroxyl protons amounts to little more than $10^{-5}$ normal, and the discovery of the structure was a fortuitous accident, since even if the ethyl alcohol used for the sample were pure to begin with, contamination of this order of magnitude could come from the solution of carbon dioxide from the air to which the sample was exposed, or even from the dissolving of acidic or basic constituents of the glass sample holders which were used when the effect was first discovered.

As was pointed out above, the disappearance of the structure of the hydroxyl resonances in the samples in which there is an excess of $\mathrm{H}^{+}$or $\mathrm{OH}^{-}$ions is attributed to a relatively rapid exchange of hydroxyl protons. It is to be expected that at intermediate rates of exchange there will be line shapes intermediate between those showing resolvable structure and those completely devoid of structure. Such intermediate cases have indeed been observed, and give thereby a means of direct measurement of rates of exchange.

From the phenomenological equations of $\mathrm{Bloch}^{11}$ it is possible to obtain an expression which predicts the line shape in a multiplet when exchange events transfer protons from one molecule to another, assuming that the effect of such an exchange is equivalent to a change of the effective fields acting upon the exchanged proton and the protons in the interacting group of the molecule in which the exchange terminates. This assumption is justified in the approximation to which Eq. (6) holds; a random change of $m_{C}$ due to exchange of the proton in the hydroxyl group is here equivalent to a change of the resonance frequency for both the methylene and the hydroxyl groups. The signal amplitude for either
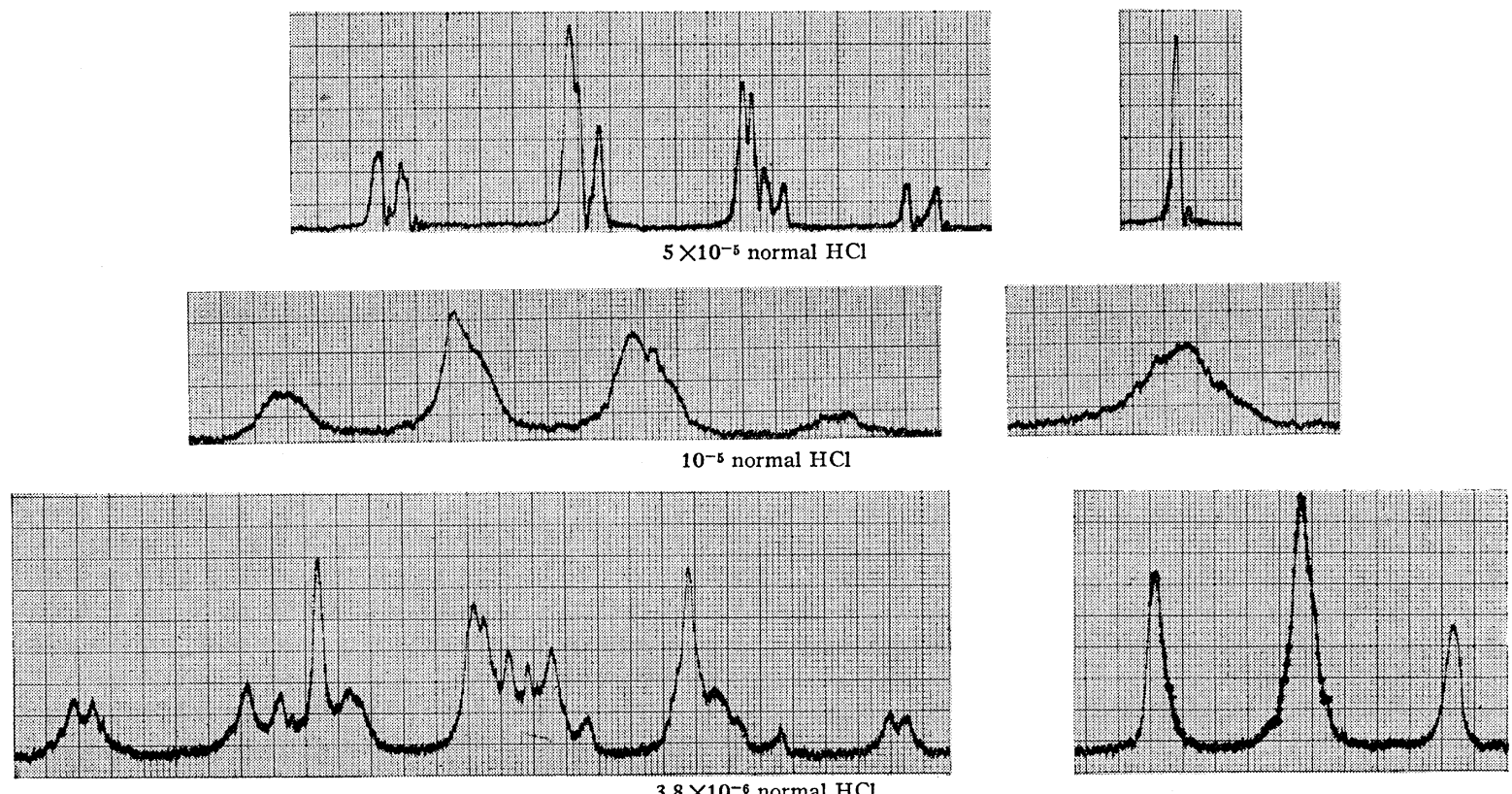

FIG. 8(a). Traces of the proton magnetic resonances of the methylene and hydroxyl groups in ethyl alcohol with varying concentration of $\mathrm{HCl}$. The zero of the concentration is arbitrary. Resolution of the apparatus is uniformly good for all three cases shown.

${ }^{25}$ J. T. Arnold, Phys. Rev. 85, 763 (1952). 
group can be derived from the general expression

$$
v+i u=-|\gamma| M_{0} H_{1} T_{2}\left\{\frac{\sum_{s} \frac{g_{s}}{1+\tau / T_{2}+i\left(\omega-\omega_{s}\right) \tau}}{\sum_{s} g_{s} \frac{1+i\left(\omega-\omega_{s}\right) T_{2}}{1+\tau / T_{2}+i\left(\omega-\omega_{s}\right) \tau}}\right\},
$$

where $\omega_{s}$ is one of the several possible values which the resonance frequency can assume within one group, $g_{s}$ the corresponding probability that the exchange will terminate in a molecule where the resonance frequency is $\omega_{s}$, and $\tau$ the mean lifetime between exchange events. In this expression it is assumed that the exchange event itself is rapid, and that the transverse relaxation times $T_{2}$ and the mean exchange times $\tau$ are independent of $s$. It is possible also to form an expression similar to (9) in which the $T_{2}$ and $\tau$ both depend on the index, $s$. From Eq. (9), line shapes may be calculated for various values of $\tau$. By a comparison of the traces obtained from samples, to which varying small amounts of $\mathrm{HCl}$ and $\mathrm{NaOH}$ have been added, with calculated line shapes, it was possible to determine the approximate value of $\tau$ for each concentration. Three typical traces for specific concentrations of $\mathrm{HCl}$ (referred to an arbitrary zero) are shown in Fig. 8(a). In Fig. 8(b), three representative calculated line shapes are given, with the exchange time $\tau$ indicated in each case.

From these experiments, it is only possible to infer the value of $\tau$ in the restricted interval where the exchange rate has a noticeable influence on the character of the resonances. This interval is defined in the case of the hydroxyl group in ethyl alcohol by the conditions

$$
\frac{\Delta \omega}{J_{B C^{2}}} \widetilde{<} \approx \frac{1}{\Delta \omega},
$$

where $\Delta \omega$ is the natural width of the resonance lines or the line width due to field inhomogeneity, whichever is larger. The values of $\tau$ found by comparison of the

TABLE II. Hydroxyl proton exchange time $\tau$ as measured for samples of ethyl alcohol, to which controlled amounts of $\mathrm{HCl}$ or $\mathrm{NaOH}$ have been added.

\begin{tabular}{ccc}
\hline $\begin{array}{c}\text { Sample } \\
\text { number }\end{array}$ & $\begin{array}{c}\text { Contamination with reference } \\
\text { to a fresh bottle }\end{array}$ & $\begin{array}{c}\text { Exchange time } \\
\tau \text { in seconds }\end{array}$ \\
\hline 1 & None & 0.24 \\
3 & $1.2 \times 10^{-6} N \mathrm{HCl}$ & 0.47 \\
4 & $1.9 \times 10^{-6} N \mathrm{HCl}$ & 1.2 \\
5 & $2.5 \times 10^{-6} N \mathrm{HCl}$ & 0.55 \\
6 & $3.1 \times 10^{-6} \mathrm{~N} \mathrm{HCl}$ & 0.6 \\
7 & $3.8 \times 10^{-6} \mathrm{~N} \mathrm{HCl}$ & 0.75 \\
8 & $4.4 \times 10^{-6} \mathrm{~N} \mathrm{HCl}$ & 0.50 \\
9 & $5 \times 10^{-6} N \mathrm{HCl}$ & 0.8 \\
13 & $7.5 \times 10^{-6} \mathrm{~N} \mathrm{HCl}$ & 1.1 \\
14 & $9 \times 10^{-6} \mathrm{~N} \mathrm{HCl}$ & 0.025 \\
11 & $10^{-5} \mathrm{~N} \mathrm{HCl}$ & 0.012 \\
& & \\
16 & & 0.3 \\
15 & $3.8 \times 10^{-6} N \mathrm{NaOH}$ & 0.006 \\
\hline \hline
\end{tabular}
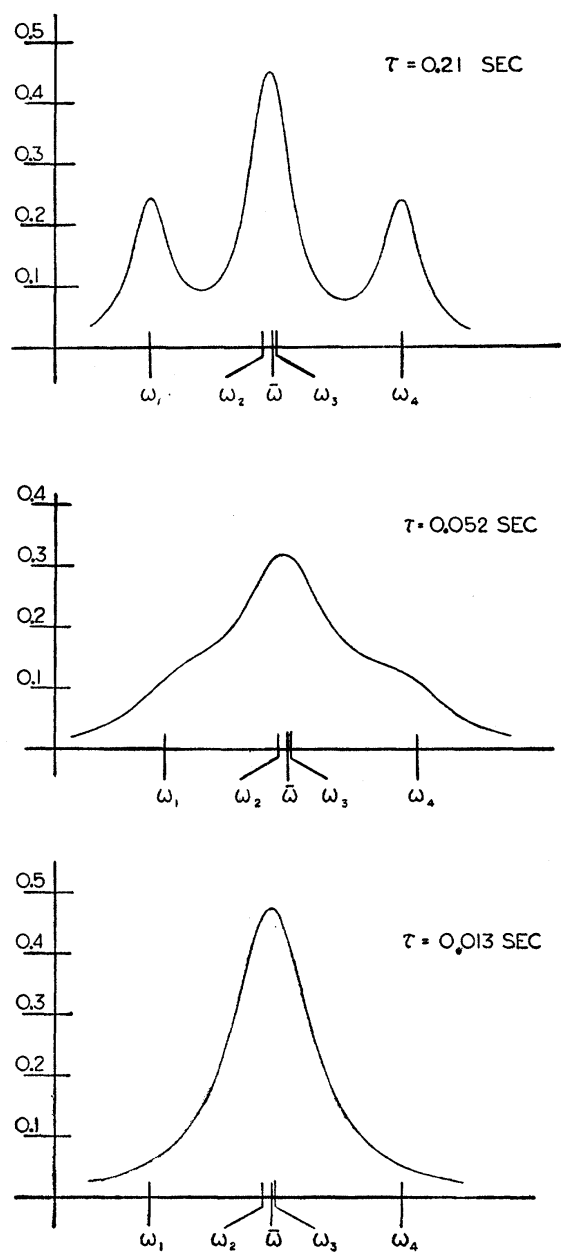

FIG. 8(b). Calculated shapes of hydroxyl group resonances in ethyl alcohol for three values of the exchange time $\tau$ on the basis of Eq. (9). The intervals $\omega_{4}-\omega_{3}, \omega_{3}-\omega_{2}$, and $\omega_{2}-\omega_{1}$ were taken in accordance with the measured value of $\tau$. $J_{B C}=4.8$ cycles per second. For all terms in the sums, $T_{2}$ was arbitrarily assigned the value of two seconds, and the $g_{s}$ were all given the value of $\frac{1}{4}$.

traces with the predicted shapes of the resonances are given in Table II for various concentrations of $\mathrm{H}^{+}$and $\mathrm{OH}^{-}$ions added to ethyl alcohol. The concentrations refer to an arbitrary zero. All samples in the series were prepared from the same bottle of stock alcohol under an atmosphere of $\mathrm{N}_{2}$. The somewhat irregular variation of $\tau$ with steadily increasing concentration of $\mathrm{HCl}$ seems to indicate that a few of the samples were improperly contaminated in spite of the precautions undertaken. For a relatively large range of concentrations, it will be noted that $\tau$ has values of the order of one second. This fact signifies that exchange broadening caused by the addition of $\mathrm{HCl}$ contributed in this range relatively little to the line width.

It is evident from these experiments that $\mathrm{H}^{+}$or $\mathrm{OH}^{-}$ ions in ethyl alcohol act as a catalyst for the exchange of hydroxyl protons between molecules, and the measurements represent in fact a determination of the 
exchange rates in a range of from one per second to about 100 per second. It is also interesting to note that the existence of structure with narrow lines in the hydroxyl group in pure ethyl alcohol indicates that the exchange rate is in this case about one per second. This fact may help to shed some light on the mechanism of "hydrogen bonding" in ethyl alcohol.

\section{CONCLUSIONS}

This high-resolution study of the proton magnetic resonance spectrum of ethyl alcohol has disclosed a large number of extremely narrow lines in a relatively small frequency interval. In this molecule, the simple Hamiltonians used in the derivation of the energies of the spin system calculated in the second and third order of spin-spin interaction lead to a system of lines which agrees remarkably well with the experimental results. It was of considerable importance to check this agreement in the case of a molecule where the chemical structure is well known in order to interpret with confidence the spectra of other molecules which may be less well understood.

That such a simple Hamiltonian should describe so completely the behavior of the spin system in a liquid is indeed remarkable, in view of the extremely complicated motions which are characteristic of liquids, and considering the fact that it ignores the actual presence of other interactions which are larger by several orders of magnitude. It is in fact the extremely frequent random interruptions of the motion of a molecule which allows the observation of delicate features of the interactions within the molecule. This is so because the random processes occur at a rate large compared to the resonance frequencies of the spin system, and, as a consequence, very effectively average out the influence of the large perturbing interactions. Neither a solid nor a diffuse gas can fulfill the requirement of rapid and complete averaging of the larger interactions during a Larmor period, with the result that line widths for these physical states are typically so broad ${ }^{26}$ as to obscure the details which are observed in the spectra of liquids. It may be useful to point out that the wellknown effect of the collision broadening of optical spectral lines represents the extreme opposite case from that of nuclear magnetic resonance in regard to the influence of random processes. The important difference lies in the fact that the optical frequencies are by contrast large compared to the rate of collisions.

The usefulness of the high-resolution techniques as a tool for the study of certain aspects of molecular structure in other organic liquids besides ethyl alcohol, and also other molecules having nuclei, other than protons, with spin one-half such as fluorine and phosphorus, is expected to increase as the methods developed to date are further improved. In particular, a more detailed study of line widths will be of great interest. Also, the application of auxiliary techniques, such as multiple irradiation of samples will be of help in the analysis of more complicated spectra of other molecules. Regarding certain chemical problems, the use of highresolution magnetic resonance techniques may be fruitful in the study of rate processes, as for example, the exchange of the hydroxyl protons described above, in a range of time intervals which has heretofore been inaccessible.

\section{ACKNOWLEDGMENTS}

The author wishes to express his gratitude to the many individuals without whose help this work could not have been carried out. In particular, he wishes to acknowledge the help and encouragement given by Professor F. Bloch and the collaboration of Dr. W. A. Anderson and Dr. M. E. Packard.

${ }^{26}$ Bloembergen, Purcell, and Pound ,Phys. Rev. 73, 679 (1948). 


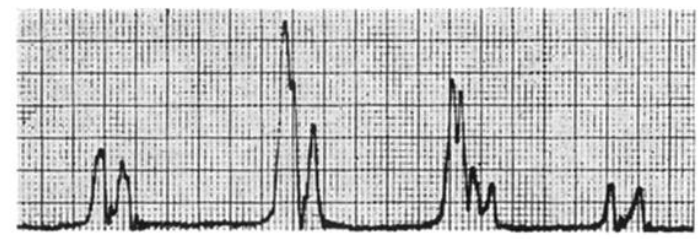

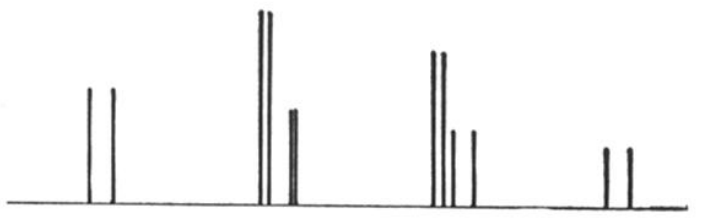

FIG. 7. High-resolution trace of the methylene group originating from impure ethyl alcohol with a diagram of the spectrum as calculated to the third order. 

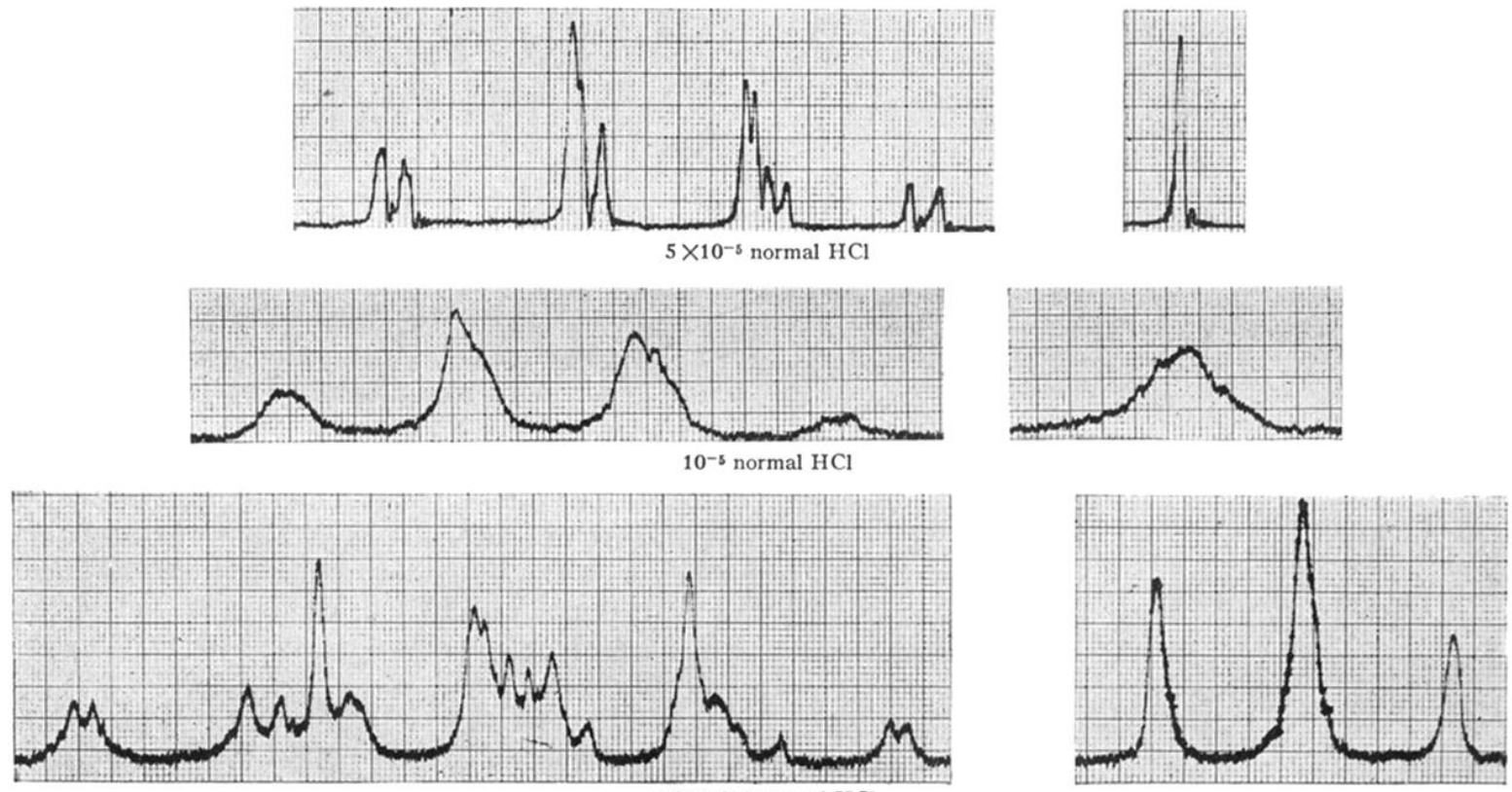

FIG. 8(a). Traces of the proton magnetic resonances of the methylene and hydroxyl groups in ethyl alcohol with varying concentration of $\mathrm{HCl}$. The zero of the concentration is arbitrary. Resolution of the apparatus is uniformly good for all three cases shown. 